Aus dem städischen St. Lazarushospital in Warschau.

\title{
Ein Fall von Hautactynomykose, mit grossen Jodkaliumdosen behandelt.
}

\author{
Von \\ Dr. Adolf Kozerski, \\ Arzt am Ambulatorium dessolben Hospitals. \\ (Hierzu Taf. IV и. V.)
}

Am 21./X. 1895 wurde auf die Abtheilung die Kranke E. W. aufgenommen. Die Diagnose lautete: Syphilis tarda. Es wurden Einreibungen von graner Salbe à $2 \cdot 0$, K.-J, $1^{*} 0$ pro die und emplastrum mercuriale auf die erkranke Partie verordnet.

Dr. v. Watraszewski, dessen Asistent ich zur Zeit war, hatte die Liebenswürdigkeit, mir den Fall zur Beobachtung zu überweisen.

Die Anamnes e ergab, dass sich die Kranke bis zum letzten Jahre der allerbesten Gesundheit erf'eute. Niemals hat sie abortirt. Vor 15 Jahren gebahr sie ein gesundes Kind, welches bis jetzt lebt und gesund ist. Das zweite, vor acht Jahren geborene Kind, erlag in der zehnten Lebenswoche der "Grippe". Das letzte, den 25./IV. 1895 geborene Kind, starb in der siebenten Lebenswoche in Folge einer hartnäckigen Diarrhoe.

Die Kranke stellt in Abrede, etwas bei sich beobachtet zu haben, woraus wir auf Syphilis schliessen könnten.

Erst im Februar 1895 bemerkte sie im Centrum der linken Wange ein Knötchen, welches mit der Zeit immer mehr an Umfang zunahm. Nach einigen Monaten, nachdem Patientin mehrmals Blutegel, Umschläge u. s. w. applicirte und der Zustand sich verschlimmerte, suchte sie Rath bei einem in der Umgebung wohnenden Arzte, der zuerst die kranke Stelle mit Zinkpaste bedecken liess, später localiter empl. cinereum und eine innere Arznei verordnete. Es trat darnach aber keine Besserung ein. Der Zustand verschlimmerte sich im Gegentheil immer mehr, es 
traten Störungen in den Kaubewegungen ein und schliesslich wurde das Fauen vollständig unmöglich, so dass die Kranke keine festen Speisen zu sich nehmen konnte.

Wir müssen noch hinzufügen, dass vor einem Monate die Patientin während einer Woche an Ohrensausen, Hals- und Kopfschmerzen gelitten hat, welche des Nachts stärker aufgetreten sein sollten.

Schliesslich hat der behandelnde Arrt sie in das St. Lazarusspital in Warschau geschickt.

Status praesens: Den 24./X. 1895 fand ich Folgendes. Fin gut gebautes 45jähriges Banernweib, mässig ernährt, von kräftigen Muskeln und gutem allgemeinem Zustande. Die sichtbaren Schleimhäute nornal. Das Linochengeriiste ohne nachweisbare Veränderungen. Die Haut. mit Ausnalmme der unten zu beschreibenden Veränderung, normal, gelblich, clastisch.

Die ganze Affection ist an der linken Wange localisirt Die Haut an derselben ist dunkelroth, cyanotisch, stark infiltrirt. Die Hyperämie und Infiltration verlieren sich gegen die Peripherie allmälig - nach oben etwas oberhaib des proc. temp. ossis zygomatici und oberhalb des Processus mastoidens. Die Ohrmuschel ist frei. Von Vorne beginnt die Grenze seitwärts von äusserem Augenwinkel, fällt dann senkrecht bis zur Nasolabialfalte, übergeht auf den Hals, wo sie $1-1 \frac{1}{2} \mathrm{Cm}$. unter der miteren Mandibulakante parallel zu derselben läntt, dann $2 \mathrm{Cm}$. liuter dem Unterkicferwinkel hebt sie sich in die Höhe, wo sie hinter der Ohrmuschel, die Haargrenze nicht erreichend, an die obere Grenze gelangt. Auf diese Weise kann die erkrankte Hautpartie mit einem gleicharmigen mit der Basis nach vorne gewendetem Dreieck verglichen werden.

Die Haut ist an der oben beschriebenen Stelle leicht verschicbbar, wit den Iinochen nicht verwachsen, glatt, glänzend, von mässig harter Consistenz, nicht schmerzhaft, hier und da mit grossen membranösen Schuppen bedeckt. Quer ungefähr durch die Mitte der Wange sind angenscheinlich die oberen Hautschichten wallartig exhaben. Der so gebildete, ungefähr 2-5 Mm. breite WuIst, läuft nach einer wellenartigen Linie, ist weich und zeigt deutliche Fluctuation. Die Sonde dringt durch einige punktförmige Oeffnungen mit Leichtigkeit in das Innere des Ganges bis zu seinen Endigungen hinein, lässt sich aber seitwärts über die sichtbaren Grenzen des Ganges nicht verschieben Nach oben und unten, fast bis zur Grenze der erkrankten Partie, lanfen in wellenartigen Windungen lïurzere Fisteln, welche mit der oben beschriebenen Fistel communiciren.

Eine längere ähnliche Fistel lüuft, ron dem untersten Punkte der Ohrmuschel beginnend, längs der unteren Kinnkante und beschreibt einen mit der Convexität nach unten gerichteten Bogen.

Schliesslich sehen wir noch eine schräg hinter der Ohrmuschel verlaufende kürzere Fistel

Die äussere Fistelwand ist augenscheinlich von oberen Hautschichten gubildet und hebt sich von der Unterlage in Form einer dünnen Membran, durch welche die darunter eingefühte Sonde deutlich durchschimmort, 
ab. Nur an denjenigen Stellen, wo Schuppen oder Krusten vorhander sind, wird die Sonde unsichtbar.

Dicker gelblichex Eiter, welcher die Fistelgänge ausfülltt und ihnen eine graugelbliche Farbe verleiht, lässt sich durch zahlreiche punktförmige Oeffnungen herauspressen. Zwischen zwei Deckgläschen lässt sich der Fiter ohne Widerstand zerquetschen. Körner sind nicht zu finden.

Bei der Untersuchung der Mundhöhle stellt sich heraus, dass fast alle Zahnkronen meistens zerstört sind. Die Bewegungen des Kinns sind derart beschränkt, dass beim stärksten Aufmachen des Mundes lie Riänder des Zainfleisches kaum um $2 \mathrm{Cm}$. von einander entfernt sind.

In der Schleimhaut der Mundhöhle lassen sich weder bei der Besichtigung, noch beim Palpiren Veränderungen nachweisen. Die zwischen zwei Fingern, von denen der eine in die Mundhöhle eingeführt wurde, betastete Wange, erweist die stärkste Infiltration in der Gegend des Kaumuskels. Die Infiltration verliert sich allmälig gegen die vordere Grenze.

Die Untersuchung der übrigen Haut sowie der inneren Organe lässt nichts abnormes nachweisen. Der Urin enthält werler Zucker noch Eiweiss. Das Körpergewicht beträgt $121 \% / 4 \mathrm{Pfd}$.

Da wir eine Eiterung des Gesichtsskeletts ausschliessen komnten, so waren wir gezwungen, den Process als eine Bildung von oberflächlichen cutanen Fisteln, welche mit Hyperämie und Infiltration der angrenzenden Haut und benachbarter Weichtheile complicirt waren, zu betrachten.

Das Krankheitsbild schien mir ausschliesslich der Actinomykose oder einer ihr verwandter parasitärer Erkrankung zu entsprechen.

Die syphilitischen Efflorescenzen (selbstverständlich ist nur von späten Formen die Rede) lassen doch immer zwei Haupttypen seines Baues erkennen, d. h. man hat vor sich ein kugeliges oder ovales Infiltrat, welches entweder in seiner Mitte (Gumma) oder an seiner Peripherie zerfällt (Ulc. serpiginosum). Das war aber in unserem Falle, wie aus der Beschreibung ersichtlich ist, keineswegs der Fall. Aus diesem Grunde fühlte ich wich berechtigt Syphilis auszuschliessen, obwohl die in der Anamnese erwähnten hartnäckigen Kopfschmerzen, die während der Nacht in stärkerem Masse auftraten, doch gewisse Zweifel über ihre Natur zuliessen.

Der Mangel an Schmerzhaftigkeit, die dicke Consistenz des Eiters, die verhältnissmässige Regelmässigkeit der Fistel ohne Tendenz sich zu grösseren Geschwüren mit unterminirten Rändern umzugestalten, endlich der Mangel irgendwelcher all- 
gemeiner Symptome - lies den Gedanken an Scrophuloderma oder irgendwelche andere Form der Hauttuberculose bei Seite legen.

Der exquisirte Charakter eines chronischen Eiterungsprocesses erlaubt uns nur der Vollständigkeit wegen an Neubildungen zu denken, um desto mehr, dass weder eine eigentliche Neubildung, noch irgend welche Characteristica (wie Epithel-Perlen bei Epithelioma) vorhanden waren.

Es unterliegt also keinem Zweifel, dass wir es in gegebenem lalle mit einer chronischen phlegmonösen Eiterung zu thm hatten. Es handelte sich also um die Lintscheidung der Crsache derselben.

Die Consistenz des Eiters war zwar in gegebenem Falle nicht für Actinomykose charakteristisch. Es wurden aber Fälle beobachtet, in welchen der Eiter bei Actinomykose nicht, wie gewöhnlich dïnn, sondern dick erschien.

Es musste natürlich die Anwesenheit von Körnern, resp. charakteristischer Pilze mikroskopisch nachgewiesen werden. Grössere Körner liessen sich aber absolut nicht auffinden, selbst nicht, nachdem ich den Eiter in dümer Schichte auf Uhrgläschen ausbreitete. Es waren zwar vereinzelte punktförmige runde Klïmpchen zu sehen, die sich aber absolut nicht von denen unterscheiden liessen, die im Eiter auderer Abstammung constatirt werden können.

In zwanzig Deckgläschen, welche mit einer dünuen Schichte aus den Fistelgängen stammendem Eiter bedeclit waren und nachdem dieselben in Kalilauge abgespült wurden und in Glycerin betrachtet waren, konnten wir keine den Actinomycesdrüsen ähnlichen Gebilde auffinden.

Diese Untersucbungen wurden mehrmals wiederholt, ergaben aber stets dieselben Resultate.

Unterdessen setzte die Kranke die Einreibungen fort, nahmin täglich $1.0 \mathrm{~K} . J$. ein und die kranke Stelle wurde mit Empl. cinereum bedeckt.

Die Trankengeschichte lautet, wie folgt:

30./ $X .1895$. Die Kranke hat 10 Inunctionen engerieben.

Die linke Wange ist deutich bliisser, die cyanotische Farbe verschwindet. 
2./XI. 1895. Die periphären Theile der Fisteln haben sich in platte weisse $3 \mathrm{Mm}$. lange Stränge umgewandelt und sind Narben inmitten einer gerötheten Haut ähnlich. Aehnliche Stränge unterbrechen die langen Fisteln ibrer ganzen Länge nach, so dass die langen Fisteln zu mehreren kürzeren getheilt erscheinen. An anderen Stellen wölbt der sich ansammelnde Eiter die Fistelwand empor.

5./XI. 16 Inunctionen eingerieben. Körpergewicht 1221/2 $\mathrm{Pfd}$.

8./XI. Zum ersten Mal färbte ich den auf einem Deckgläschen ausgebreiteten Eiter nach Gram. Unter zahireichen polynucleären Leukocyten (nachgefärbt mit Vesurin) fand ich einen Filz von Fäden, deren Beschreibung weiter unten folgen wird. Die Fäden waren theilweise in grösseren Haufen verfilzt, theilweise einzeln in der nächsten Umgebung des Filzes zerstreut.

Nachdem noch einige andere Deckgläschen dasselbe Resultat ergaben, nämlich charakteristische Actinomycesfäden, wurde die Diagnose : "Actinomykosis faciei" erwiesen.

Ich schlug die von Thomassin an Thieren und von van Iterson an Menschen erprobte Jodkaliumtherapie um desto mehr vor, da die Erfahrungen, welche Meunier, Buzzi, ${ }^{1}$ ) Poncet, Ramsom, ${ }^{2}$ ) Rydygier, $\left.{ }^{3}\right)$ Pringle') und Andere gesammelt haben, nur dazu anregten. Die Inunctionen und das graue Pflaster wurden bei Seite gelegt.

Ich möchte an dieser Stelle erwähnen, dass während der gemischterı antiluetischen $\mathrm{Cur}$ einige Besserung eingetreten ist. Die Infiltration der Wange verschwand, die eyanotische Gesichtsfarbe hat einer mehr röthlichen Platz gemacht. Die periphären Fistelpartien waren allem Anschein nach in Heilung begriffen. Die Kranke war im Stande, den Mund etwas breiter aufzumachen.

Da mir Anfang's noch kein photographischer Apparat zu Gebote stand, habe ich erst nachträglich den 8./XI. die Kranke photographirt. (Fig. 1.)

8./XI. Kalii jodati 4.0 de die in zwei Einzeldosen.

9./XI. Mein Vorschlag, einen Zahn zur Untersuchung herauszuziehen. wurde von der Kranken abgelehnt. KJ 4.0 de die.

10./XI. KJ 4.0 de die. 11./XI. Körpergewicht 1191/2 Pfd. KJ 6.0 de die. 12./XI. KJ 6.0 d. d. 13./XI. KJ 8.0 d. d. 14./XI. Die Kranke verträgt sehr gut KJ. KJ 8.0 d. d. 15./XI. KJ 10.0 d. d.

16. XI. In der rechten Achselhöhle haben sich einige kleine folliculäre Abscesse gebildet. Der entleerte Eiter enthält weder Körnchen, noch Actinomycesfäden. KJ 12.0 d. d.

1) Choux. Étude clinique et therapeut. de l'Actinomycose. Archives générales de médecine. 1895 p. 687.

$\left.{ }^{2}\right)$ Centralblatt für Chirurgie. 1894, 1895. Referate.

$\left.{ }^{3}\right) \mathrm{V}$. Congress der polnischen Chirurgen.

4) Monatshefte für prakt. Dermatologie. 1895 p. 235. 
17./XI. Die Besserung des Zustandes ist mit jedem Tage deutlicher. Die Infiltration ist viel kleiner, der Mund lässt sich immer breiter aufmachen. Unterdessen machen die Fisteln alle früher beschriebenen Stadien durch. Es sammelt sich nämlich der Eiter und zwar augenscheinlich viel. rascher als früher. Die Fistelwand wölbt sich und platzt an ihrer dünnsten Stelle. Der daraus hervortretende Eiter ist immer von sehr dicker Consistenz, enthält winzig kleine weisshiche Körnchen, die erst deutlicher auftreten, nachdem man den Eiter in dünner Schichte ausbreitet. Nach der Entleerung des Eiters fallen die Fistelwände zusammen, lassen sich noch eine gewisse Zeit über einander verschieben, was aber schon nach einer Woche misslingt. Dann verwandeln sich die Fisteln in einen weissen narbigen Strang, der mit der Zeit immer enger und kürzer wird. K.J 12.0 d. d.'

18. XI. Appetit, Digestion gut. Der allgemeine Zustand lässt nichts zu. wünschen übrig. - Körpergewicht 123 Pfd. KJ 12.0 d. d.

19./XI. Man sieht im Eiter Körnchen, deren die grösseren einen Durchmesser von $1 / 2 \mathrm{Mrn}$. erreichen. KJ $14.0 \mathrm{~d}$. d.

20./XI. KJ 14.0 d. d.

21./XI. Keine Reizerscheinungen seitens der Schleimhäute, die auf Jodvergiftung hindeuten möchten. KJ 14.0 d. d.

22./XI. Die Kranke ist im Stande immer grössere Excursionen mit dem Unterkiefer zu Stande zu bringen. KJ 14.0 d. d.

23. XI. Kopfschmerz. Schnupfen. KJ 0.

24./XI. Der Schnupfen ist geringer. Der Kopfschmerz ist fast verschwunden. KJ 0 .

25. XI. Schnupfen und Koyfschmerz vollständig geschwunden. Es bestand keine Sicherheit, dass der Schnupfen dem Jodgebrauch zuzuschreiben wäre. Es fehlte Reizung weder der Speicheldrüsen, noch derjenigen Schleimhäute, die gewöhnlich die ersten an Jod reagiren. Die Secretion. der Nasenschleimhaut war auch nicht so reichlich, wie es gewöhnlich. bei Jodschnupfen der Fall ist. Deswegen bestand keine Sicherheit, dass der Schnupfen dem Jodgebrauch zuzuschreiben wäre. Es lag viel näher. an gewöhnliche Schädlichkeiten zu denken, von denen in Spitälern die Kranken kaum zu schützen sind, wie Erkältungen in schlecht beheizten Aborten, Herumgehen mit nackten Füssen auf dem feuchten Boden der Waschstube u. s. w. - In Folge dessen, nachdem 24 Stunden weder Schnupfen, noch Kopfschmerz zu verzeichnen waren, kehrten wir zu einer kleineren Dosis Jodkalium 8.0 pro die zurück.

26. XI. KJJ $9.0 \mathrm{pe}$ d.

27. XI. Körpergewicht $128 \frac{1}{2}$ lPfd. College A. Z Zurakowski hat die Freundlichkeit gehabt, einige kleine Körnchen auf Agar zu überimpfen. - KJ $9.0 \mathrm{~d}$. d.

28./XI. Es bleibt nur ein intracntaner Abscess an der Stelle der sonst zusammengefallenen längsten Fistel. KJ 10.0 p. d.

29. XI. Die ganze linke Wange ist fast vollständig frei von Infiltration und blassrötblich gefärbt. An der Stelle der früleren Fisteln sieht 
man weisse flache Stränge, die theilweise allen Anschein einer Narbe haben, sind hart, durch eine in ihr Inneres eingeführte Nadel der Prav a z'schen Spritze lassen sie sich nicht mit Flüssigkeit füllen. - Die anderen wieder, obwohl sie auch als weisse Narbenstränge imponiren, lassen sich doch etwas über ihrer Unterlage verschieben. Die Prava z'sche Spritze füllt sie noch mit Flüssigkeit, doch nur an bestimmten beschränkten Bezirken. Es macht den Eindruck, dass der früher in seiner ganzen Länge durchgängige Canal jetzt in mehreren Punkten obliterirt ist. KJ $10.0 \mathrm{p}$. d. verheilt.

30./XI. KJ 10.0 p. d. Die Abscesse in der Achselhöhle sind

1./XII. Kopfschmerz, Schnupfen. KJ 0.

2./XII. Status idem. Körpergewicht $i 28 \frac{3}{4}$ Pfd. KJ 0. - Natrik salicylici 0.5 zweimal täglich.

3./XII. Kein Kopfschmerz. Der Schnupfen ist geringer. KJ 0.

4./XII. Kein Schnupfen mehr. KJ 4.0 p. d. 5./XII. KJ 8.0 p. d. 6./XII. KJ 8.0 p. d. 7./XII. KJ 8.0 p. d. $8 . /$ XII. KJ 8.0 p. d. 9./XII. Körpergewicht $128 \frac{1}{2}$ Pfd. KJ 9.0 p. d. 10./XII. KJ 9.0 p. d.

11./XII. Die Infiltration der Wange ist vollständig verschwunden. Geringe Ueberreste derselben sind noch unmittelbar vor, unter und hinter der Ohrmuschel zu sehen. Die Unterkieferbewegungen sind vollständig frei. Die Mund- und Rachenschleimhaut ist normal, blass, ohne Narben. Die Kranke hört etwas schlechter mit dem linken Ohr, als mit dem rechten, was aber nach ihren Angaben noch vor dem Beginne der Krankheit sich einstellte. Der linke äussere Gehörgang ist eng, lässt nur das engste Speculum durch. So viel man Einsicht bekommen konnte, war die Membrana tympani als blasse weisse glänzende Membran zu sehen. KJ wurde wegen der wieder eintretenden Kopfschmerzen nicht gegeben. täglich.

12./XII. Schnupfen, Kopfschmerz. Natrii salicylici 0.5 zweima]

14. XII. Der Schnupfen ist geringer, der Kopfschmerz hat aufgehört. 16./XII. Körpergewicht $132 \mathrm{Pfd}$. 17./XII. Der Schnupfen ist fast verschwunden.

18./XII. Die rosarothe dünne linke Wange ist frei von Eiterherden. Nur hinter der Ohrmuschel in der Gegend des Processus Mastoideus ist eine runde flach infiltrirte Hautstelle von $23 \mathrm{Mm}$. Duchmesser zu sehen, dessen Mitte ein kleiner Schorf bedeckt.

19./XII. Der Schnupfen ist verschwunden.

21./XII. Abends starker Schüttelfrost. Vom linken äusseren Gehörgang fliesst dünnflüssiger Eiter mit weissen Klümpfchen heraus, die unter. dem Mikroskope als aus Eiter- und Epithelzellen gebildet sich herausstellen. Von den Mikroorganismen sind zahlreiche Coccen von verscbiedener Grösse zu sehen, aber keine Actinomycesfäden. T. 38.1.

22./XII. Morgentemperatur 37.1. Abendtemperatur 37.6.

23./XII. MT 37.0. AT 37.6. Der Eiter, der jetzt in bedeutender Menge nicht nur aus dem äusseren Gehörgang, sondern auch durch die Tuba Eustachii fliesst, ist trüb und dick geworden. (Otitis media sup- 
purativa.) Die Kranke gurgelt mit Kalium chloricum und trockene Wattebäuschchen mit Borsäure werden in den Gehörgang eingelegt und oft gewechselt.

24./XII. MT 37.1. AT 37.3. Der mikroskopische Befund des immer reichlich herausfliessenden Eiters ist immer derselbe. Immer sind Actinomyycesfäden nicht zu finden. Die linke Rachenhälfte ist stark geschwollen and geröthet. Schmerzen beim Schlucken.

25./XII. MT 31.0. Der Ausluss ist weniger stark. Die Schmerzen beim Schlucken sind geringer. Nachmittags starker Schüttelfrost. P 39.6. Die Hant vor, unter und hinter der linken Obrmuschel stark geröthet, geschwollen, heiss, schmerzhaft beim Betasten, scharf von der gesunden Partie abgegrenzt. Diagnosis: "Erysipelas," Es wurden local Compressen von: Hydrarg. bichlor. corrorivi 0.1, Aluminis acetici 1.0, Aq. destil. 100.0 applicirt.

26./XII. T 37.8. Die Hyperämie und Schwellung hat sich nicht nur über das ganze früher durch die Actinomykose eingenommene Territorium verbreitet, sondern ist auch auf die benachbarten Partien übergegangen, so, dass die linke Gesichtshälfte unterhalb des Processus zygomatici und der Augenspalte ist von einer heissen, stark rothen, gespannten, glänzenden, schmerzhaften Haut bedeckt. AT 38.7.

27/XII. Die Entzündung ist auf beide Augenlider und auf die Nase über ihre Medianlinie übergegangen. Aus dem äusseren Gehörgang fliesst wenig Eiter heraus. Die Röthe der linken Rachenlälfte ist viel geringer. MT 37,3; AT 37.9.

28. XII. Die entzündliche Schwellung ist anf die rechte Regio zygomaticae übergegangen. Die linke Gesichtshälfte ist hart infiltrirt und cyanotisch verfärbt. Innerhalb einiger Fisteln, nämlich derjenigen, welche am spätesten ihren eitrigen Inhalt verloren haben, tauchen citrige Punkte auf. MT 36.8. AT 37.9.

29./XII. Beide Lider des linken Auges sind kolossal geschwollen, erlauben nicht das Auge aufzumachen. Im oberen hari infiltrirten Augenlid fühlt man einen fluctuirenden Abscess. Die Grenze der entzündlichen schmerzhaften Schwellung nähert sich immer mehr und mehr der rechten Ohrmuschel. MT 379. AT 38.3.

30. XII. Seit zwei Tagen Stuhlverstopfung. In dem aus dem Abseess des Augenlides entnommenen Eiter liessen sich keine Actinomycesfäden nachweisen. Auch auf der rechten Gesichtshälfte macht die lebhafte Röthe einem blaulichen Ton Platz. Nur die äusserste Grenze der in Entzündung begriffenen Partie, welche jetzt bis zur rechten Ohrmuschel reicht, hat noch den lebhaft rothen Ton behalten. MT 39.6, AT 38.1. 0 . rycini i Esslöffel.

31./XII. Die linke Gesichtshälfte ist viel blässer geworden, die Schwellung hat sich auch bedentend zurückgebildet. Aus der im oberen Augenlide befindlichen Abscesshöhle fliesst viel dicker Eiter, welcher lseine Actinomyceselemente enthält. Die rechte, bläulich gefärbte Gesichts hälfte ist ebenfalls stark abgeschwellt. Die Entzündung ist über die Ohr- 
muschel hinüber gewandert, ohne jedoch die Haargrenze zu überschreiten. MT 38.3. A'T 38.8. Chinini muriatici 0.2 zweimal täglich.

1./. 1896. Die Schwellung ist sowohl ron der linken, wie von der rechten Gesichtshälfte vollständig verschwunden. Die rechte Gesichtshälfte ist noch rosaroth verfärbt, die linke von normaler Hautfarbe, desquamirt. $\mathrm{MTT}$ 37.0. AT 36.5.

2./I. An der rechten Gesichtshälfte lässt sich kaum ein leicht rother Hauch walınehmen. Stuhlverstopfung. Nach einem Eisslöffel Ricinusöl 6 flüs ige Stühle. Bismuti subnitrici $1.0 \mathrm{zweimal}$ täglich. MT 36.8. AT 37.2. Chinin wurde ausgesetzt.

3./I. MT 35.8. Puls schwach, 60 in der Minute. Kräfteverfall. AT 35.9. Wein. Kräftige Diät. Coffeini 0.1 zweimal täglich.

4./. Die Kranke fühlt sich besser. MT 35.8. P 68. AT 36.2. P 70, etwas stärker.

5.I. MT 37.0. AT 36.9. Puls Morgens 72, Abends 70. Die Kranke ist lebhafter.

6./I MT 36.1. P 70. AT 36.4. P 72. Körpergewicht 123 Pfd. Die letzten Ueberbleibsel der Röthe sind verschwunden. Aus dem linken Gehörgang fliesst melir Eiter heraus.

7./. MT 36.0. AT 36.9. Appetit gut. Die untere Partie der linken Wange ist wieder geröthet und angeschwollen. Eisbeutel auf die geschwollene Partie.

8.I. MT 36.2. AT 36.9.

9. I. MT 36.5. AT 36.8. Die Infiltration der unteren Partie der linken Wange wird bedentender. Druck auf diese Stelle verursacht keine Schmerzen.

10./I. Die Körpertemperatur ist immer unter 37.8. Innerhalb der infiltrirten cyanotisch-rothen stelle, an der Stelle einer der früberen Fisteln, sind einige kleine Abscesse zu sehen. Die Haut ist hinter der linken Ohrmuschel vollständig normal.

13./I. Körpergewicht $126 \mathrm{Pfd}$.

14./I. Der Ausluss aus dem Ohreanal beschränkt sich auf ein unbedeutendes Quantum.

15./. Einige Stellen in der Gegend des aufsteigenden Kieferastes zeigen Fluctuationsgefühl. Ein Einstich fordert einen Tropfen Blut heraus.

16./I. Herr College A. Żurakowski hat wieder die Güte gehabt die Körnchen aus den oben erwähnten, bis jetzt noch nicht eingestochenen kleinen Hautabscessen sowie den Eiter ans dem linken Gehörgang auf Agar zu überimpfen. Während der ganzen Nacht sammelt sich auf dem Wattebäuschchen im linken Ohr kaum ein kleiner Tropfen Eiter.

17./I. In der Nacht einige flüssige Stüble.

18.I. Wieder einige flüssige Stühle in der Nacht. Bismuthi subnitriei 1.0 Abends.

19./I. Nachts einige flüssige Stühle. Natrii bromati 1.0 Abends.

20.I. Nachts Diarrhoe. Tinct. opii Gtt. X. Abends. 
21./I. Keine Diarrhoe. Nach vorne vom unteren Ende der linken Ohrmuschel bildete sich ein kleiner Hautabscess von der Grösse eines Weizenkorns aus.

22.I. Aus dem gestern erwähnten Abscess nach dessen Einstechung habe ich dicken Eiter mit zahlreichen kleinen weisslichen Körnchen grewonnen.

23./I. Die Einstichstelle ist mit einer trockenen Kruste bedeckt.

25./I. Unter der Kruste sammelt sich kein Eiter mehr.

28./I. Hinter dem linken Ohr haben sich zwei kleine Abscesse, den vorigen der Grösse nach gleich, ausgebildet. In dem entleerten Fiter: findet sich eine Menge von Körnern von noch nicht gesehener Grösse; einige von ihnen erreichen die Grösse eines Mohnlkorns.

31 /. In der Mitte des aufsteigenden Kieferastes sieht man einen r'unden Schorf von der Grösse eines halben Weizenkorns. Nach Abnehmen desselben zeigt sich eine kleine Quantität Eiter mit vielen Körnchen. 8 von ihnen habe ich auf Glicerinagar überimpft und habe Herrn Collegen A. $\dot{Z}$ u rakowski übersandt.

$5 /$ II. Hinter der Ohrmuschel hat sich wieder ein kleiner intracutaner Abscess ausgebildet.

7./II. Unterhalb der Ohrmuschel sieht man wieder 3 kleine Abscesse mit vielen Körnern. Aus dem linken Gehörgang entleert sich kein Witer. Wir sind wieder zuKJ zurückgekommen, und zwar wïhlten wir den Weg per anum. Ich habe mich nämlich mehrmals überzeugt, dass KJ manclimal von solchen Kranken auf diese Weise vertragen wird, bei welchen das per os applicirte sofort Schnupfen erzeugt hat. Die Kranke bekam KJ 2.0 pro die.

10./II. Keine Jodvergiftungssy mptome. Appetit normal. Körpergewicht $128 \mathrm{Pfd}$.

11./I. Der Allgemeinzustand gut. Keine Fitersecretion aus dem linken Gehörgang. Durch das enge Speculum sieht man die stark hyperämische injicirte Membrana tympani. Die Kranke hört mit dem linken Ohr weniger, als mit dem rechten. Die Kieferbewegungen sind vollständig frei. Die Schleimhaut des Rachens und der Mundhöhle ist blass. Die Speichelsecretion nicht übermässig stark. Die rechte Wange normal. Die Haut der linken Wange an dem Bezirke, welcher frïher von der detinomykose eingenommen war, ist von normaler Farbe, vielleicht ctwas pigmentirt, brännlich, von normaler Dicke, in der unteren hinteren Partie etwas infiltrirt, überall leicht über der Unterlage verschieblich. Als Ueberbleibsel der früheren Fisteln sind weisse barte 1-2 Mm. breite Streifen zurückgeblieben, die das Aussehen von Narben haben. Die Streifen sind viel enger und kürzer, als die Fisteln, axs welchen sie entstanden sind.

Der Heilungsprocess ist an denjenigen Stellen am weitesten vorgeschritten, an welchen er am frühesten begonnen hat, nämlich die Nurben sind schon in Schrumpfung begriffen. Nur in 3 Punkten, nämlich: 2 unter her Ohrmuschel, 1 in der Gegend des Collum Processes condyloidei mandibulae sielat man kleine runde firusten. 
Nachdem ich die liranke plotographirt hatte (Fig. 2.), habe ich der Reihe nach in eine jede gewesene Fistel die Nadel der mit Wasser gefüllten Prava z'schen' Spritze eingestochen und versucht, die vermuthliche Fistel mit Wasser zu injiciren, was mir aber überall misslang. Ich halte also die weissen Streifen, wie es mir scheint mit Recht, für wahre Narben. Nas unter die zuletztgenannte liruste injicirte Wasser füllte die von ilır ausgehenden weissen Streifen auf der Strecke von $5 \mathrm{Mm}$. beiderseits. Doch ertrielt ich keine Körner aus dieser Stelle.

12./II. 1896. Die Kranke verliess das Spital auf eigehes Verlangen. Sie sollte sich auch zu Hause 2.0 Jodkali per anum täglich einführen.

Nach sechs Wochen besuchte die Kranke wieder das Spital. Am ganzen Bezirke, der früher von uns als geheilt anerkannt wurde, ist kein Fiterherd zu sehen. An den Stellen, welche bei der Entlassung der Kranken aus dem Hospital mit Krusten bedeckt waren, bestehen dieselben noch. Nach ihrer Aufhebung lässt sich kaum etwas Eiter gewinnen, der einige Körnchen enthält. Es erwies sich, dass die Kranke uur so viel Medicin eingenommen hat, bis die ihr im Spital gegebene Portion ansreichte. Als sie aber genöthigt war sich dieselbe für eigenes Geld zu schaffen, konnte sie sich dazu nicht entschliessen. Auf diese Weise hat sie nur die ersten fünf Tage Jodkalium eingenommen, ist also während mehr als 5 Wochen ohne Behandlung geblieben.

Die Notiz, welche mir Herr College A. '̇urakowski zu übersenden die Güte hatte, lantet wie folgt: "Nach der üblichen Desinfection der Haut der Wange wurden die aus dem Fisteleiter gewonnenen Körner auf Agar übertragen, womach die Eprouvetten in den 'Termostat bei T. 37, ${ }^{\circ}$ C. eingestellt wurden. Nach einer Woche sah man schon die Körnchen an Umfang zunehmen; sie waren doch immer noch einem Schleimtropfen ähnlich. Erst am Ende der zweiten Woche hat die Oberfläche eines solchen Tropfens ein mehr charakteristisches Aussehen gewonnen, ist weisslich, wie ausgetrocknet geworden und bedeckte sich mit Runzeln. Die Ueberimpfung der Körner gelang jedesmal. Da aber solche Reincultur sogar nach sechs Wochen sehr unbedeutend wuchs, habe ich sie ohne Sauerstoffzutritt zu cultiviren versucht. Der Versuch ist glücklich gelungen, die Cultur begann nämlich üppiger zu wachsen und ist noch jetzt beim Leben erhalten. Es ist noch zu bemerken, dass gleich rom Eiter die Reincultur gewonnen wurde. 
Der Versuch, die Cultur aus dem Eiter zu der Zeit zu bekommen, als die Cur sich an ihr Ende näherte, wurde auch von positivem Erfolg begleitet.

Vom Eiter aus dem linken Gehörgang erhielt man nur den Staphylococcus albus und aureus."

Wie gesagt, untersuchte ich die ersten Präparate des Fisteleiters in ungefärbtem Zustande in Glycerin, nachdem sie vorher in $2 \%$ Kalilauge abgespült waren. Obwohl ich immer nur ganz frische Präparate zur Untersuchung nahm, enthielten sie absolut nichts, was irgendwelche Aehnlichkeit mit bekannten Actinomycesdrusen verrieth. In Präparaten, welche nach der VanGie s o n'schen Methode gefärbt wurden, ausser sehr zahlreichen weissen, meist polynucleären Blutkörperchen waren hie und da grosse runde Körper zu sehen, die eine starke gelbrothe Farbe annahmen. Es scheint mir, dass dies eben die Körnchen waren, in welchen später die Gram'sche Methode die Actinomycesconvoluten entdeckte.

Das erste nach Gram gefärbte, mit Vesurin nachgefärbte Präparat, gab folgendes Bild.

Unter einer Menge weisser Blutkörperchen zeichnen sich runde, stärker mit Vesuvin sich färbende Gebilde, innerhalb welcher schon unter AA Zeiss Ocular 2 eine feine dunkelblaue Zeichnung wahrnelimbar ist, aus. Unter Immersion $1 / 12$ stellt sich heraus, dass wir mit einem dichten Convolut dunkelblau gefärbter Fäden zu thun haben, die zwischen den Eiterkörperchen eingelagert sind. (Fig. 3.) In den dichtesten Stellen des Convoluts kann man kaum die einzelnen Fäden unterscheiden. Dies ist nur am Rande des Convoluts möglich und dort, wo die Füden wahrscheinlich beim Zerquetschen des Präparates vom Convolut abgerissen wurden.

Die Fäden, homogen gebaut, zeigen oft eine gabelförmige Theilung. Viele haben an ihren Endigungen eine ovale längliche dunkel gefärbte, $1 \%$ Mal breitere, als der Faden selbst, Verdickung, die unmittelbar vom Fadenkörper entsteht, ohne von ihm auf irgendwelche Weise abgegrenzt zu sein. Andere Fäden sind an beiden Enden dunkel gefärbt, eiförmig verdickt. Andere wieder bilden lange Ketten, als ob sie auf dem Wege der Gliederung des einzelnen Fadens entstanden wären. Nicht 
alle sind gleich intensiv gefärbt. Meist sind die dickeren dünkler. Manche haben in ihrem Ende oder in dem. Mittelstück weniger Farbstoff zurückgehalten. Wenige tragen in ihrem unverdickten Ende einen dunklen Punkt.

Dasselbe Bild wiederholt sich auch in anderen Präparaten, welche aus der ersten Periode der Beobachtung stammen. Wir begegnen hier alle Gestalien, von Körnchen angefangen, deren Durchmesser demjenigen der Fäden gleich ist, anderen etwas längeren, bis zu wohl ausgebildeten einzelnen und gabelförmig sich theilenden Fäden mit quer abgeschnittenen unverdickten oder geschwollenen dünkleren Enden.

Bei der Untersuchung des von den Fäden gebildeten Filzes lässt sich absolut keine Regel in seinem Baue auffinden. Das makroskopische Körnchen, zwischen zwei Deckgläschen zerquetscht, chne dieselben übereinander zu ziehen, cingetrocknet und nach Gram gefärbt, erimnert mit seiner Structur mehr an einen Schwamm, als einen Stern. Deun zwischen den Streifen des Filzes, der dem Chitingerüste des Schwammes analog wäre, sind freie Canäle zu sehen, die wieder den Schwammcanälen entsprechen.

Im Präparate, welches aus dem Eiter den 6./XII. 1895 (Fig.4) bereitet wurde, fand ich zurm ersten Mal eine grössere Quantität der Fäden, die etwas anders, als die bisher beschriebenen, gebildet waren. Nämlich einige, schwach gefärbt, enthalten in ihrem Inneren eine ganze Reihe Körnchen, die verschiedene Grösse und Intensität der Färbung aufweisen. Sie färben sich doch immer intensiver, als wie der Faden, in welchem sie sich befinden. Die anderen Elemente des Präparates waren den früher beschriebenen identisch.

Es wäre noch zu erwähnen, dass aus je späterem Stadium der Beobachtung der zu untersuchende Eiter stammte, desto häufiger begegnete ich bei der Untersuchung die Körnchen enthaltende Fäden, so, dass z. B. im Präparate, welches vom 15./XII. stammt, findet man zahlreiche Conglomerate von Fäden, die gänzlich in Körnchen zerfallen sind, wobei in einem die Fadencontouren obwohl schwach, doch noch zu sehen sind, während in anderen nur die in Rosenkränze gereihten Körnchen ihre Entstehung aus Fäden beweisen. 
Den 2./I. 1896 bekam ich zur Untersuchung einen Theil der Reincultur, welche Herr College A. Zurakowski gezüchtet hat. Zu diesem Zweck zerquetschte ich einen kleinen Theil der Cultur zwischen zwei Deckgläschen, zog dieselben von einander $a b$, trocknete auf der Luft, fixirte durch mehrmaliges Durchziehen durch die Spiritusflamme, färbte in warmem Anilinwassergentianaviolett und im Uebrigen behandelte ich sie nach der Gram'schen Methode. Die Untersuchung der Präparate hat folgendes Resultat ergeben. (Fig. 5 u. 6.)

Als Grundform begegnet man immer einem dichten Filz, welcher von einzelnen Fäden zusammengewebt ist. Die Fäden sind entweder homogen, oder kettenweise gegliedert, oder in kleine runde dunkel gefärbte Körnchen zerfallen; sie sind einfach oder gabelförmig getheilt; mit ovalen Verdickungen an den Enden, oder ohne denselben; sie sind entweder in ihrer ganzen Länge gleich intensir gefärbt, oder die Endigungen oder die Mitte sind mehr mit Farbstoff gesättigt. Neben sehr langen findet man ganz kurze. Manche tragen in ihrer Mitte eine spindelförmige Verdickung, die mehr den Farbstoff aufnimmt. Zwischen den Fäden sielıt man zahlreiche Körnchen, die entweder in Rosenkränze gereiht sirid, oder ohne irgendwelche Anordnung in Haufen oder zerstreut liegen. Man findet auch ovale Bruchstuicke, die mit den oben beschriebenen Verdickungen der Fäden ganz identisch zu sein scheinen, die jedoch ohne Zusammenhang mit den Fäden frei liegen. Endlich babe ich dreimal in Trauben zusammengehäufte runde Gebilde gefunden, die weniger intensir gefärbt und grösser sind, als die bisher beschriebenen mikroskopischen Körnchen, viel kleiner aber, als die beschriebenen ovalen Gebilde.

Was die Entstehung der Körnchen aus den Fäden anbelangt, so ist man kaum im Stande zu bestimmen, in welchem Theile des Fadens die Körnchen hauptsächlich gebildet werden. Man findet die Körnchen ebenso häufig in kurzen, wie in langen Fäden; in einem Theile der letzteren, oder in ihrer ganzen Länge, sowohl im Fadenleibe, wie in ihren Verdickungen.

Die meisten Fäden zerfallen in eine einfache Reihe von Körnchen. Es gibt aber auch solche, nach welchem allem Anscheine nach eine doppelte Reihe ron Körnchen zurückbleibt. 
Innerhalb anderer wieder finden wir die Körnchen nach einer wellenartigen Linie gelagert, wie Kugeln in einer etwas zu breiten Rinne. Am seltensten sind solche, in welchen auf ein stärker gefärbtes Glied immer ein ganz blasses folgt.

Wenn wir dieses Bild mit demjenigen rergleichen, welches uns die Untersuchung des Eiters ergab, so sehen wir, dass die Elemente in beiden Fällen dieselben sind. Der einzige Unterschied besteht darin, dass in der Reincultur eine viel grössere Anzahl der Fäden in Körnchen zerfallen ist.

Der Vollständigkeit wegen sei hier noch erwähnt, dass weder in der Reincultur, noch im Präparate vom Eiter irgendwelche andere Mikroorganismen gefunden wurden. Die oft wiederholte Untersuchung des Eiters, welcher aus dem linken Ohr floss, ergab kein einziges Mal Actinomyceselemente. Das zuletzt erwähnte Eiter enthielt kleine und grosse Coccen, zuweilen in Ketten, meistens aber zu zwei oder in Haufen gelagert.

Dasselbe Resultat ergab die Untersuchung des Eiters, welcher aus dem Abscesse des Oberlides stammte.

Da die Kranke sich entschieden vor einer Excision eines Stückchens der erkrankten Haut weigerte, so konnte der Fall histologisch nicht untersucht werden.

Nach dem oben gesagten braucht nicht mehr bewiesen zu werden, dass wir mit einer Mykose zu thun gehabt haben, die eine chronische Eiterung in der Haut hervorgerufen hat.

Das stete Auffinden im Eiter der Pilzelemente, welche denjenigen des Actinomyces vollständig gleichen ${ }^{1}$ ) und mit anderen Eiterung hervorratenden Pilzen morphologisch nichts gemeinsames haben, hat mir genug Grund gegeben, mit Recht Actinomykosis zu diagnosticiren.

Es ist wahr, dass wir kein einziges Mal den so charakteristischen Bestandtheil des Actinomycespilzes - die Kolben - auffinden konnten, obwohl wir sehr viele Präparate in frischem Zustande untersuchten. Doch wird der Umstand kaum gegen die Diagnose sprechen angesichts der Meinung Boströ m's, die immer mehr Anbänger gewinnt, dass die Kolben nur ein

') Birch-Hirschfeld. Real-Encyklopädie, 189t. Bd. I p. 227. 
Degenerationsproduct des Actinomycespilzes sind und in Folge dessen seinen am mindesten wichtigen Antheil bilden.

Noch weniger könnte die dicke Consistenz des Eiters, die vor nicht langer Zeit als der Actinomykose widersprechend galt, die Diagnose erschüttern, da sie doch bereits in vielen citirten Fällen reiner Actinomykose beobachtet wurde.

Es bliebe noch die Möglichkeit, dass wir es mit einem Fall von Pseudoactinomykose zu thun hatten ähnlich denjenigen, welche Hesse, Rosenbach, Rabe und Andere beschrieben haben. Doch da der von uns in gegebenem Falle gefundene Pilz alle wesentlichen Characteristica des Actinomyces besitzt, finde ich keinen Grund an andere Parasiten zu denken.

So möchte ich also die Diagnose der Actinomykose in gegebenem Falle als sicher bewiesen ansehen.

Es lässt sich nicht dasselbe über die Complication sagen, welche den 25./XII. sich dazugesellt hat. Für die Diagnose "Erysipelas" möchten folgende Gründe sprechen: der einzige starke Schüttelfrost, die Spannung und Schmerzhaftigkeit der erkrankten Haut, die scharfe Abgrenzung derselben von gesunden Partien. Endlich fehlte es auch nicht an Gelegenheit zur Infection.

Denn auf das nächststehende Bett wurde eine aus der Stadt ankommende Kranke mit Ulcus cruris installirt, bei welcher an demselben Abend ein starker Schüttelfrost ein typisches Erysipelas einleitete, dem auch sehr hohe Temperatur nicht fehlte. Und obgleich die letzte Kranke sofort separirt und der Platz nach ihr desinficirt wurde, so war doch die Zeit, während welcher die Kranken nebeneinander lagen, ausreichend, um das Erysipel auf unsere Kranke zu übertragen.

Was aber gegen die Diagnose "Erysipelas" sprechen möchte und eher an eine Phlegmone denken liesse, das ist die niedrige Temperatur, welche nur zweimal $39.6 \mathrm{C}$. gelang und sonst in normalen Grenzen sich hielt.

Auf die Frage, durch welche Pforte der Parasit in den Organismus eingedrungen ist, lässt sich kaum eine ausreichende Antwort geben. Der häufigste Weg, d. h. durch einen cariösen Zahn, lässt sich hier nicht ausschliessen, da alle Zähne ca- 
riös sind. Es blieb jedoch keine Spur z. B. in Form eines narbigen Stranges, welcher die erkrankte Hautpartie mit dem Perioste des Kiefers verbinde, zurück. Die Kranke kann sich auch nicht erinnern, dass jemals die Gegend eines Zahnes geschwollen und schmerzhaft gewesen wäre. Sie betont im Gegentheil mit Bestimmtheit, dass die Krankheit ganz oberflächlich in der Mitte der Wange begann.

Der Sitz des Parasites ist ausschliesslich in der Haut gewesen, denn obwohl die Infiltration auch die tiefer liegenden Gewebe einnahm, so verliefen doch die Fisteln ausschliesslich in der Haut und während der ganzen Beobachtung konnte man keine Eiterung oder Fluctuation in tieferen Geweben bemerken. Das möchte uns vielleicht gewissermassen berechtigen, eine Infection durch die Haut zu vermuthen. Doch obwohl ähnliche Fälle wohl bekannt sind, ${ }^{1}$ ) es fehlt uns die absolute Sicherheit, um unseren Fall zu derselben Kategorie einzureihen. Wir beschränken uns also, unseren Fall als Hautactinomykose zu bezeichnen, indem wir damit nur die Localisation des Processes meinen.

Bei der Application des JK hatten wir zwei Wege zur Einführung des Medicamentes zu wählen. Nämlich entweder durch den Verdauungscanal, oder mittelst Injectionen. (Die Applicationsweise, welche Gautier mit Erfolg anwand und welche darin besteht, dass das injicirte KJ mittelst Elektrolyse zerspaltet wird, war in unseren Verhältnissen zu umständlich.) Wir liessen bei Seite die Injectionen, und zwar aus folgenden Gründen.

Rydygier injicirte in die Substanz des Tumors. Wir haben aber vor uns keinen Tumor gehabt. Die Fliissigkeit, welche wir in die Fisteln injicirt haben, floss sofort durch zahlreiche Oeffnungen hinaus; nicht einmal der Eiter wurde dadurch ausgespült. Es war keine Möglichkeit vorhanden, das Jodkalium in den Fisteln zuriickzuhalten. Häufig wiederholte Injectionen unter die Gesichtshaut könnte unangenehme Complicationen hervorrufen. Aus diesem Grunde wendeten wir uns zu erster Applicationsweise.

1) Leser. Centralbl. f. Chir. 1889. Nr. 29. Choux 1. c. p. 573 u. 585. 
Als wir bemerkten, dass je grössere Dosis applicirt wurde, desto schneller der Heilungsprocess verlief, steigerten wir immer die Dosis.

Die Beobachtung des Verlaufes in unserem Falle lässt sich in Folgendem resumiren:

Die mehr als seit 8 Monaten an Actinomykose leidende Frau, so wie unbehandelt, kommt ins Spital ohne irgendwelche Spuren der spontanen Heilung. Nach 16 Inunctionen und 16.0 KJ (1.0 pro die) lässt sich einige Besserung bemerken. Nämlich die Hyperämie und Infiltration der Wange werden geringer, die Kaubewegungen gewinnen etwas an Ausdehnung und die peripheren Fisteltheile verlieren ihren eitrigen Inhalt.

Dann während 31 Tage (mit 5tägiger Pause) nimmt die Kranke KJ ein in Dosen von 4.0 bis 14.0 pro die. Während dieser Zeit verschwinden die Infiltration der Wange und der Trismus vollständig. An der Stelle trüherer Fisteln entstehen Narben. In drei Stellen vor und unter der linken Ohrmuschel die Reste der Fisteln lassen sich noch in einer Ausdehnung von $1 \mathrm{Cm}$, mit Flüssigkeit füllen, doch ist jede sichtbare Literung in ihnen exloschen. Es bleibt noch ein einziger kleiner Eiterherd hinter der Ohrmuschel.

Als Folge der Invasion durch den linken Gehörgang der eitererregenden Mikroorganismen wird wieder die Wange in acuter Weise hyperämisch und infiltrirt. Es bilden sich $\mathrm{Ab}$ scesse, aber nicht an den Stellen, welche wir als geheilt ansahen. Der Eiter enthält keine Elemente des Actinomyces. Nur in drei Punkten um die untere Partie der linken Ohrmuschel, wo nicht vollständig geheilte Ueberreste der Fisteln zurückgeblieben sind, kommt unter dem Einflusse der durch die Complication verursachten Hyperämie zu Eiterung und dieser Eiter enthält Actinomyceselemente.

Als die Complication rorbei war, bekommt die Kranke wieder das Jodkaliuu 2.0 pro die per anum während sechs Tagen, wornach trotz unseres Rathes sie das Spital mit drei Punkten, die mit kleinen Krusten bedeckt sind, verlässt.

Zn Hause nimmt sie nur fünf Tage lang das KJ ein, die übrigen fünf Wochen bleibt sie ohne Behandlung. Kommt dann 
wieder ins Spital zurück ohne Recidiv in geheilten Stellen. Nur dieselben drei Krusten, die auch beim Verlassen des Spitals an denselben Stellen sichtbar waren, beweisen, dass der krankhafte Process an dieser Stelle noch nicht erloschen ist.

Wenn wir nun fragen, welche die Ursache einer so bedeutenden Besserung während unserer Beobachtungszeit war, so können wir vor allem meiner Ansicht nach die Inunctionen bei Seite legen. Ihre geringe Zahl und der bis jetzt meines Wissens nicht beobachtete Einfluss derselben auf den Verlauf der Actinomykose lässt ihren Einfluss mindestens bezweifeln. Die locale Behandlung der erkrankten Stelle mit grauem Pflaster, der während erster 16 Tage applicirt wurde, konnte ohne Zweifel einen gewissen positiven Einfluss auf die Heilung ausüben. Stau $b^{\mathbf{1}}$ ) hat die Gelegenheit gehabt, einen wohlthuenden Einfluss der Ichthyol-, Chrysarobin- und Resorcinpflaster in zwei Fällen zu constatiren, worunter in einem die chirurgische Behandlung fehlschlug. Es ist wahr, dass in dem ersten Falle Staub gleichzeitig auch KJ applicirte, welches vielleicht mehr die Heilung bewirke, als der Pflaster, obwohl Staub die entgegengesetzte Meinung ausspricht. Doch im zweiten Falle der positive Erfolg, besonders in älteren Herden, war auch ohne Jodkaliumgebrauch zu constatiren.

Deswegen geben wir zu, dass der graue Pflaster auch in unserem Falle einen gewissen Antheil am Heilungsprocess genommen hat. Doch erstreckt sich wahrscheinlich seine Wirkung nicht über seine Anwendungszeit hinaus.

Was absolut ohne heilenden Einfluss auf die Actinomykose geblieben ist, das ist die Complication, welche innerhalb der Beobachtung eingetreten ist. Im Gegentheil, wahrscheinlich in Folge der acuten Hyperämie, die Infiltration um den Winkel des Unterkiefers plötzlich steigerte und so zeigte sich wieder Eiterung in diesen Stellen, welche keinen Eiter mehr führten.

Der wohlthuende Einfluss des KJ scheint hier ausser jedem Zweifel zu stehen. Es tödtete nicht den Actinomycespilz $a b$, was leicht verständlich ist, wenn man die Resistenz-

1) Therapeutische Monatshefte. 1894. Nr. 10 p. 499. 
kraft des Parasites berücksichtigt. Es gedeiht ja die Actinomycescultur auf Gelatine, welcher grössere Quantitäten KJ zugesetzt wurden, wie es die Versuche des Prof. d'Alfort beweisen. ${ }^{1}$ ) Uebrigens die gelungenen Culturen ror und zu Ende der Behandlung beweisen genug, dass der Pilz seine vitale Kraft nicht eingebüsst hat. $\mathrm{Ob}$ seine Lebensfähigkeit mindestens theilweise abgeschwächt war, lässt sich bei der Schwierigkeit, mit welcher er sich künstlich cultiviren lässt, kaum beweisen. Wenn wir aber im Auge behalten, dass die grosse Mehrzahl der Eiterherde definitiv geheilt wurde, und dass die consecutiven Krankheitserscheinungen, wie Hyperämie, Infiltration und Eiterung verschwunden sind, so ist es schwer, die Vermuthung abzulehnen, dass doch die Anwesenheit des JK im Organismus ein weniger geeignetes Nährmaterial für den Actinomycespilz gebildet hat.

Uebrigens kann doch die Frage, auf welche Weise das Jodkalium auf den Actinomyces wirkt, nicht im mindesten die klinische Erfahrung erschüttern. Denn es wäre uns gleichgiltig, ob Actinomyces durch Kalium jodatum abgetödtet wird, oder nicht, wenn wir nur wissen, dass er auf die Dauer seine Folgen aufhebt. Wir handeln ja nicht anders bei der Behandlung der: Lues tarda, obwohl wir nicht wissen, ob und unter welchen Bedingungen das KJ die vermeintlichen Syphilisbacillen zu remichten im Stande ist. Doch wenden wir es an, weil es die Symptome beseitigt.

Was die Dosirung anbelangt, so ist es sehr wahrscheinlich, dass auch kleinere Gaben ausreichen möchten und würden weniger gefährlich sein. Denn, obwohl wir noch weit von der Maximaldosis entfernt waren, die gegen andere Hautleiden, wie z. B. Psoriasis, anempfohlen wurden, ist doch fast sicher, dass die zu hohen Jodkaliumdosen in gegebenem Falle eine starke Reizung und Steigerung der Secretion der Nasenschleimhaut und in der bereits nicht vollständig gesunden linken Tube Eustachii herbeigeführt haben und den Einbruch der eitererregenden Bakterien erleichterten. Vielleicht konnte man es rermeiden, wenn wir das Jodkalium vom Anfange gleich per rectum eingeführt hätten.

l) Choux lo c. 
Zum Schluss möchte ich folgende Sätze hervorheben, die in unserer Beobachtung ihre Bestätigung finden. Nämlich:

1. Bei reiner Infection mit dem Actinomycespilze ohne 'Theilnahme der gewöhnlichen eitererregenden Bakterien ist der Eiter, welcher dabei secernirt wird, nicht immer dünnflüssiger Consistenz.

2. Der Actinomycespilz ist im Stande allein, ohne Beimischung der gewöhnlichen eitererregenden Mikroorganismen, Eiterung hervorzurufen.

3. Die Einwanderung der gewöhnlichen eitererregenden Mikroorganismen in die Herde der Actinomykose tödtet nicht den Pilz derselben ab; im Gegentheil, es können Bedingungen durch dieselben hervorgerufen werden, welche die Entwicklung des Actinomycespilzes begünstigen (mindestens, wenn die Körpertemperatur dabei nicht erhöht bleibt).

4. Die mikroskopische Untersuchung mittelst Methoden, nach 'welchen der Actinomycespilz nicht gefärbt wird, kann in manchen Fällen der Actinomykose unzureichend sein.

5. In Fällen, in welchen die Diagnose zwischen Actinomykose und Syphilis tarda schwankt, ist es unzulässig, die Diagnose ex jurantibus zu stellen, besonders, wenn KJ zur Behandlung gebraucht wurde.

6. Angesichts der wachsenden Zahl der Fälle, in welchen das JK entweder definitive Heilung oder bedeutende Besserung verursachte, wäre zweckmässig, in jedem Falle von Actinomykose mit dieser Behandlungsmethode zu beginnen.

Zum Schluss sei mir gestattet, meinem hochverehrten Chef, Herrn Med. Dr. K. v. Watras zew ski, für die freundliche Anvertrauung des Falles, Herrn Collegen A. Zu urakowski für die bereitwillige Uebernahme des bakteriologischen Theiles und dem Studenten der philosophischen Facultät, Herrn K. Kujawski, für die Ausführung mikroskopischer Photographien meinen verbindlichsten Dank auszusprechen. 


\section{Irklärung der Abbildungen auf Tafel IV u. $V$.}

Fig. 1. Nach der Photographie, die den 8. Nov. 1895, das heisst im Beginne der Behandlung, aufgenommen wurde.

Fig. 2. Nach der Photographie, die den 11. Feber 1896, d. h. am Tage der Entlassung der Kranken aus dem Spital, aufgenommen wurde.

Fig. 3. Photographie des Filzes, welchen der Actinomycespilz im Eiter bildet. Der Eiter wurde den 8. Nov. 1895 ans der Fistel genommen. Vergrösserung $1: 1000$.

Fig. 4. Die Photographie des Präparates des Eiters vom 6. Dec. 1895. Vergrösserung $1: 500$.

Fig. 5. und 6. Photographien der Reineultur. Vergröss. 1:1000 und $1: 500$. 

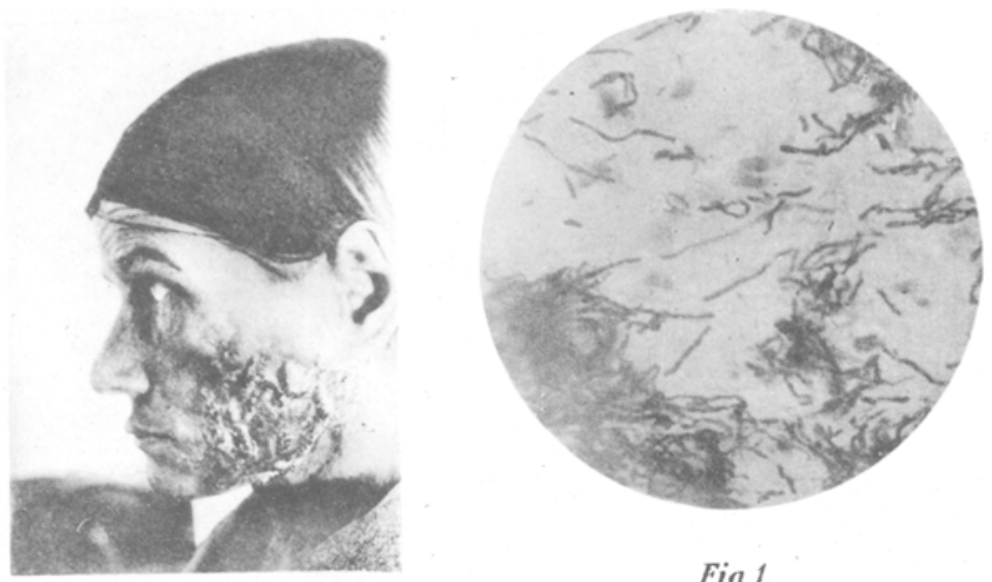

Fig 1.
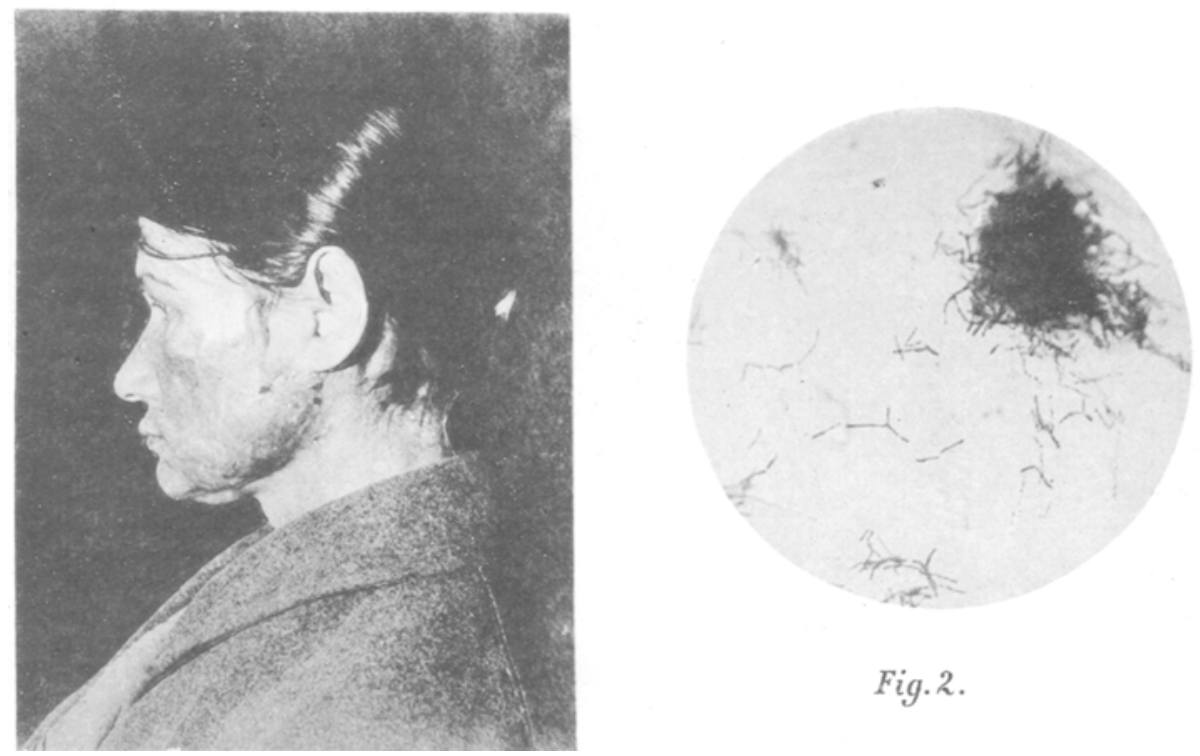

Fig. 2.

Kozerski: Hautactinomycose etc. 


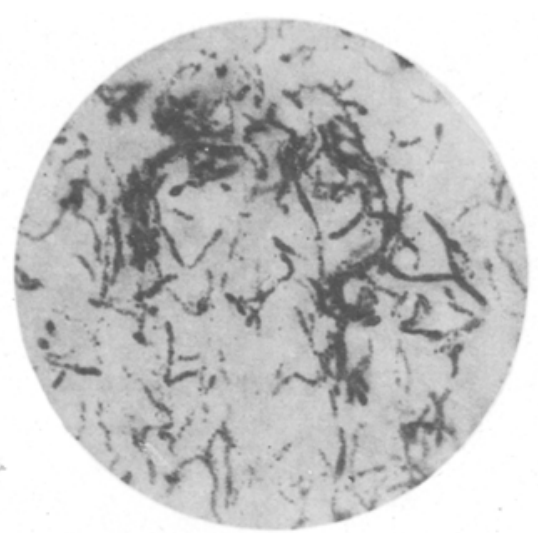

Fig.3.

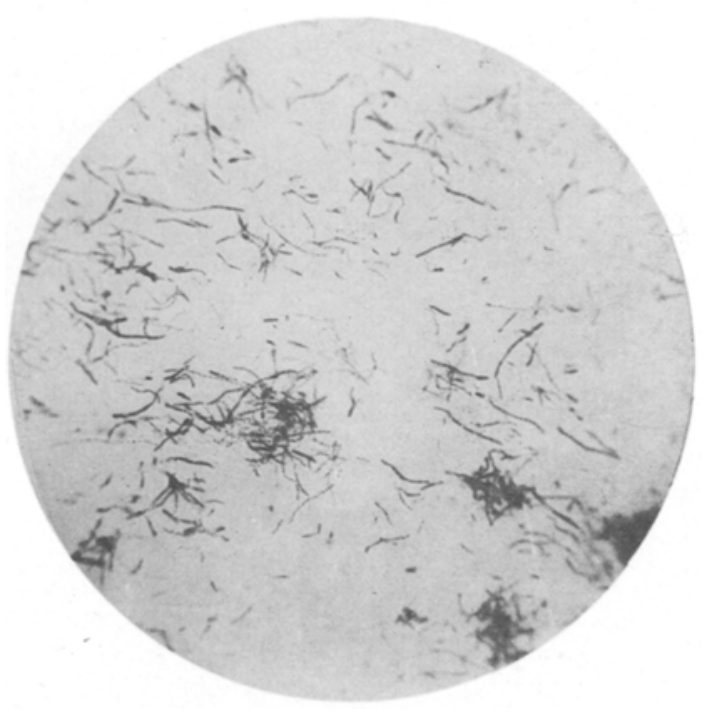

Kozerski : Hautactinomycose etc.

Fig. 4 . 